\title{
Editorial: Towards Future Research on Space Weather Drivers
}

\author{
Hebe Cremades $^{1}$ (D) Teresa Nieves-Chinchilla $^{2}$ (D) Cristina H. Mandrini $^{3}$
}

Accepted: 22 October 2021 / Published online: 16 November 2021

(C) The Author(s), under exclusive licence to Springer Nature B.V. 2021

The workshop "Towards Future Research on Space Weather Drivers" brought together 120 heliophysicists from the international community. The workshop was held in the city of San Juan, Argentina, from 2 to 9 July 2019, with the kick-off of the meeting being marked by the total solar eclipse on 2 July. The total solar eclipse was visible within a band $\approx 200 \mathrm{~km}$ wide that spanned the contiguous Argentina and Chile from well into the Pacific Ocean up to the Atlantic coast. The workshop participants witnessed the event from the "Valle Encantado", a region about $115 \mathrm{~km}$ to the north of the meeting site. The marvelous view of the eclipsed Sun over the Andes mountains, encompassed by the amazing landscape, in which attendees were immersed, offered an unforgettable experience.

The central goal of the meeting was to promote the discussion and exchange of new knowledge in the field of space weather, compelling for our modern technology-reliant society needs, from the point of view of the phenomena that modulate it from their origin in the Sun, through their evolution in the interplanetary medium, until their arrival to geospace. The topics addressed at the meeting included the latest findings on phenomena and structures affecting space weather, from the solar interior to the lower solar atmosphere, energy release in the low atmosphere, initiation and consequences of eruptive phenomena, coronal mass ejections (CMEs), solar energetic particles, radio emissions, and solar radiation variation of short time-scale; this added to coronal large-scale structuring and coupling with the solar wind, interplanetary space weather drivers, tools and simulations for predictions, and future missions and instrumentation with space weather applications. The importance of the topics discussed during the meeting envisions the advances for the improvement of space weather forecasts based on their drivers, provided by recent and upcoming cuttingedge space-based missions and new solar instrumentation that was designed to detect and

This article belongs to the Topical Collection:

Towards Future Research on Space Weather Drivers

Guest Editors: Hebe Cremades and Teresa Nieves-Chinchilla

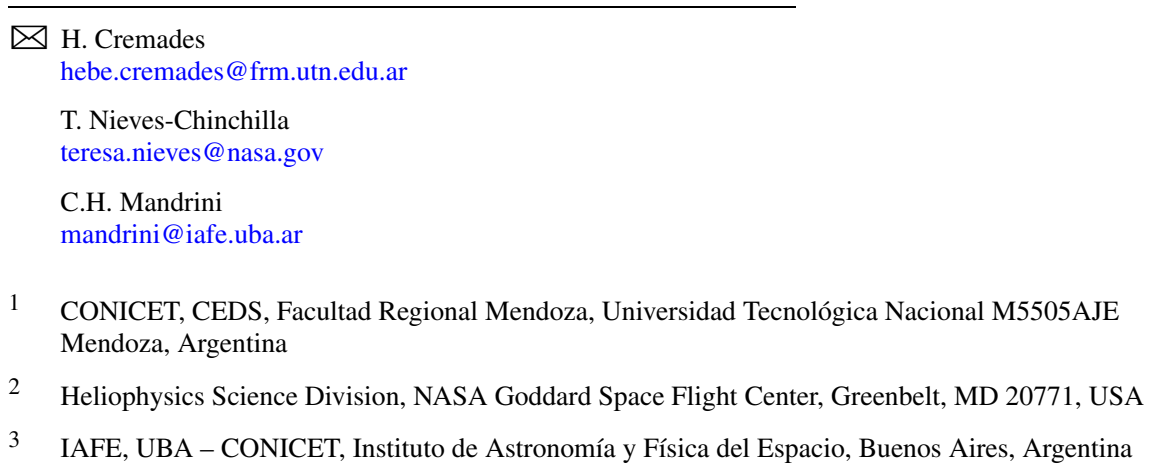




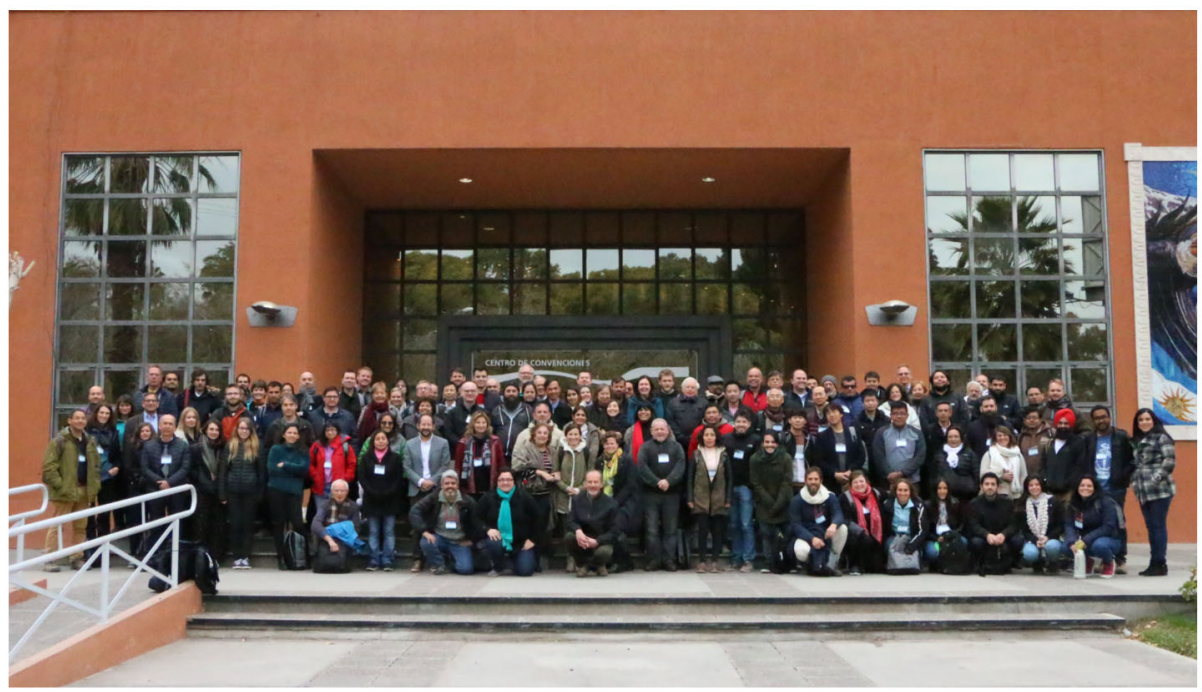

Figure 1 Group photo taken in front of the Convention Center "Intendente Guillermo Barrena Guzmán" during the workshop.

probe solar and interplanetary phenomena, together with increasingly more realistic simulations. This is the motivation behind this Solar Physics Topical Collection (TC) of 21 articles that covers such a broad range of subjects of interest to a large portion of the scientific community. Figure 1 shows the group of researchers from all over the globe that attended the workshop.

The future of space exploration is clearly determined by the space weather forecast capabilities. Among others, solar flares are the most powerful events in the solar atmosphere with a sudden release of energy, radiation, and energetic particles. The impact on planets, space technology, or human exploration may take just a few minutes, and, therefore, any progress in the prediction of their occurrence and magnitude is extremely important. The TC contains three articles that directly address this topic. On one hand, one of them includes a fundamental investigation of the physical processes associated with solar flare precursors (Hudson, 2020), and, on the other, the remaining two are applied investigations toward forecasting applications (Morales and Santos, 2020; Cinto et al., 2020). Following the previous studies, Hudson (2020) systematically searched for the evidence of a build-up and release pattern of flare-CME behavior in the 14 largest active regions of Solar Cycle 24. The research by Morales and Santos (2020) revisited the Lu and Hamilton avalanche model for solar flares in order to explore its predicting capabilities for large events. The promising results of this research suggest that these avalanche models are suitable for exploring forecasting capabilities and pave the way to implement new features in the future. The research published by Cinto et al. (2020) proposed a novel methodology for designing flare predictors, validated with extreme gradient boosting tree classifiers and time series. This methodology relies on a machine learning-based pipeline that is validated by three proof-of-concept models for forecasting flares with an X-class larger than $\mathrm{C}$ up to $72 \mathrm{~h}$ ahead. These models are able to increase significantly the scores of true skill statistics (TSS) under different operational forecasting scenarios. This methodology also leads to an improvement of the positive and negative recalls of classifiers, while decreasing the number of false alarms. 
The solar atmosphere is the region where solar flares or CMEs are energized and, therefore, of great relevance not only for space weather purposes but also for the understanding of the Sun-Earth environment. In addition, the featured coronal holes and sunspots determine the solar cycle and the three-dimensional (3D) heliospheric magnetic configuration that establishes the Sun-heliosphere connection. In this respect, within this collection, fundamental investigations have been performed to understand the solar atmosphere plasma thermodynamics, magnetic field configuration, and long-term variations (Lloveras et al., 2020; Ryabov and Vrublevskis, 2020; Kostyuchenko and Bruevich, 2021; Nandy, 2021). Lloveras et al. (2020) carried out, compared, and reported a detailed study of the global $3 \mathrm{D}$ structure of the electron density and temperature of the quiescent inner solar corona based on the differential emission measure tomography (DEMT) and the Alfvén Wave Solar Model (AWSoM) in their latest versions. They reported on the presence of loops with temperature decreasing/increasing with height (dubbed down/up loops) in agreement with previous DEMT studies. These authors also estimated the heating energy flux required at the coronal base to keep these structures stable, found to be of the order of $10^{5} \mathrm{erg} \mathrm{cm}^{-2} \mathrm{~s}^{-1}$. Due to the importance in determining the field line connectivity and topology with resulting consequences for both plasma confinement and dynamics, Ryabov and Vrublevskis (2020) used the Potential Field Source Surface (PFSS) model and revealed new details in the strong fields of the dominant sunspot of the active region (AR) 8535. Two main questions drive this investigation: 1) What is the magnetic structure of a sunspot in which a lane of reduced coronal emission goes across it? 2) Does the revealed magnetic structure allow plasma to outflow to the heliosphere? These questions are also important for the investigations related to the long-term variation of sunspots. This is another noteworthy contribution to this TC. In the study carried out by Kostyuchenko and Bruevich (2021), the authors focused on the fine structure of quasi-biennal oscillations (QBOs) of the sunspot areas during Solar Cycles $19-23$. The QBO period gradually decreases from the beginning of the solar cycle till the end in phase with the shift of the average latitudes of sunspots to the equator: a scenario of this behavior within the convection zone is proposed. Nandy (2021) (Editor's choice) reviewed the predictions for Sunspot Cycles 24-25 from different groups based on diverse techniques and performed a comparative analysis of these predictions. The analysis reveals that while predictions based on diverse techniques continue to disagree, physical modelbased forecasts for Solar Cycle 25 have converged, indicating a weak to moderate-weak sunspot cycle. Finally, in connection with the heliosphere, El-Borie et al. (2020) examined the interconnection and phase asynchrony between the periodicities of solar wind parameters and the interplanetary magnetic field (IMF) polarity during the period of 1967-2018, i.e. five solar cycles. The study reveals positive correlations and long-term to mid-term periodicities during most of the entire period of study.

The stream interaction regions created by the interaction of fast solar wind streams originating in solar coronal holes (CHs) with slower solar wind can be also a trigger of geomagnetic activity, even in lower layers of the Earth's magnetosphere. The study carried out by Palacios et al. (2020) (Editor's choice) sheds light on the understanding of the evolution of coronal holes. Specifically, in this article, the authors illustrated the evolution of the size of a coronal hole observed on 24 September 2017 during the joint-campaign observations of the Japanese Hinode mission, the GREGOR solar telescope, and the Vacuum Tower Telescope (VTT). The study carried out by Molina et al. (2020) analyzes the solar-heliosphericmagnetospheric-ionospheric processes of an intense geomagnetic storm produced in the period of 2-7 October 2015 that was driven by a corotating interaction region (CIR). This TC also brings the description of the High Altitude THz Solar Photometer (HATS), a full-Sun ground-based telescope designed to observe the continuum from the submillimeter to the 
mid-IR (Giménez de Castro et al., 2020). This article described the telescope characteristics and its development status as well as estimates of the expected fluxes during flares.

Heliospheric observations of type II and III radio emissions are critical sources of information of transient dynamics. One of the contributions to this collection from Melnik et al. (2021) relates the properties of type III and type IIIb bursts in the frequency band of $8-80 \mathrm{MHz}$ observed by the radio telescopes Ukrainian Radio Interferometer of NASU2 (URAN-2, Poltava) and Giant Ukrainian Radio Telescope (GURT, Kharkiv) during the Parker Solar Probe (PSP) perihelion in April 2019. The corona is characterized through the analysis of drift rates and duration dependencies on frequency for these bursts. More specific to interplanetary shock-wave transients, Aguilar-Rodriguez and Corona-Romero (2020) analyzed type II radio emissions observed by the Wind/Waves instrument to determine the emission region in shock fronts. In this article they used the blast wave reconstruction technique. The results suggest that the processes of emission of a type II radio burst occur not only in the upstream region of the shock, but also that there must be emission of the material that has been compressed as well as of the material that is in the compression region within the structure of the shock itself. In relation to other large-scale transients, i.e. CMEs, Shanmugaraju et al. (2021) predicted the occurrence rate of radio-loud (associated with type II radio bursts) and halo CMEs in Solar Cycle 25 and obtained good correlations between their numbers in each year and the yearly mean sunspot number in the previous two cycles.

Solar energetic particles are accelerated in solar flares, while interplanetary shocks associated with CMEs may also spread them in the heliosphere. Thus, in this TC, the number of studies, from the more fundamental to applied space weather ones, devote their investigations to CMEs. Chertok (2020) analyzed the relationship between the propagation transit time of CMEs and interplanetary CMEs (ICMEs) from the Sun to the Earth and the maximum intensity of their geomagnetic storms (isolated, non-recurrent) as measured by the geomagnetic index Dst. Out of a total of 140 events, two groups were considered on the basis of the source region type, i.e. active region and filament eruptions from quiescent areas of the Sun. Events in the first group are characterized by a shorter transit time and much stronger geomagnetic storms in comparison with the events from quiescent regions. The obtained results mean that both the speed of CMEs/ICMEs and the strength of the magnetic field transported by them are largely determined by the parameters of the corresponding eruptions, i.e. the eruptive magnetic flux and the released energy. Other very common in situ signatures of ICMEs are Forbush decreases (FDs), i.e. short-term reductions in the galactic cosmic ray (GCR) flux. Dumbović et al. (2020) applied modeling to different ICME regions to obtain a generic two-step FD profile that qualitatively agreed with current observation-based understanding of FDs. Models were adapted for energy dependence to enable comparison with GCR measurements from different instruments. Comparison of a set of multi-spacecraft observations of the same event with the Forbush decrease model for the expanding flux rope (ForbMod) yielded reasonable agreement, indicating that modeled FDs reflect well the CME evolution.

CME evolution and propagation in the heliosphere is still one of the critical areas of research. In this collection, there are several studies that span from a case event analysis, data analysis, 3D simulation, theoretical understanding of the physical processes associated with the evolution, and identification of the internal structure based on artificial intelligence techniques (Davies et al., 2020; Balmaceda et al., 2020; Desai et al., 2020; Florido-Llinas, Nieves-Chinchilla, and Linton, 2020; dos Santos et al., 2020). As stated by Balmaceda et al. (2020) (Editor's choice), a proper characterization of the kinematics of CMEs is important not only for practical purposes, i.e. space weather forecasting, but also for the improvement of our current understanding of the physics behind their evolution in the middle corona and 
the heliosphere. Thus, their study focused on the observations obtained with COR2 onboard the Solar Terrestrial Relations Observatory (STEREO) from 2007 to 2014 to investigate the self-similarity assumption as a function of the heliocentric distance of the CME events. Davies et al. (2020) reported the case of a multipoint-observed magnetic cloud by the Advanced Composition Explorer (ACE), Wind, the Acceleration, Reconnection, Turbulence and Electrodynamics of the Moon's Interaction with the Sun (ARTEMIS), and Juno satellites on 25 October 2011. This singular opportunity allowed the exploration of the radial and longitudinal variation of the internal properties of the magnetic cloud. Desai et al. (2020) described 3D magnetohydrodynamic simulations of the inner heliosphere leading up to and including the extreme ICME of 23 July 2012; the simulation used the code PLUTO. Their results suggest that the ICME was mostly unaffected by prior events, but drag forces due to solar wind preconditioning played a significant role in the propagation in the inner heliosphere. The TC also includes a novel theoretical approach to advance in the understanding of the physical processes associated with CME evolution, in this case, the twisted fields in the Sun's atmosphere. Florido-Llinas, Nieves-Chinchilla, and Linton (2020) (Editor's choice) aimed to present a theoretical study of the conditions under which magnetic flux ropes with different twist profiles are kink stable and therefore amenable to remain radially propagating. Finally, another novel approach to understand the internal structure of ICMEs based on machine learning techniques is also presented in this issue. dos Santos et al. (2020) aimed to identify and classify the flux rope signatures found in the internal magnetic structures of ICMEs. This model presents a strong potential to evolve into a robust tool for identifying flux rope configurations from in situ data.

All in all, although broad in nature, the subjects of this Topical Collection address many recent advances in the field of Heliophysics, having as overarching research aim to advance knowledge on the drivers of space weather. We hope the community finds these articles useful and worth considering when pursuing forthcoming investigations.

\section{References}

Aguilar-Rodriguez, E., Corona-Romero, P.: 2020, On the emission region of type II radio bursts in interplanetary shock fronts. Solar Phys. 295(6), 77. DOI. ADS.

Balmaceda, L.A., Vourlidas, A., Stenborg, G., St. Cyr, O.C.: 2020, On the expansion speed of coronal mass ejections: implications for self-similar evolution. Solar Phys. 295(8), 107. DOI. ADS.

Chertok, I.M.: 2020, On the relationship between the transit time of ICMEs and strength of the initiated geomagnetic storms. Solar Phys. 295(6), 74. DOI. ADS.

Cinto, T., Gradvohl, A.L.S., Coelho, G.P., da Silva, A.E.A.: 2020, Solar flare forecasting using time series and extreme gradient boosting ensembles. Solar Phys. 295(7), 93. DOI. ADS.

Davies, E.E., Forsyth, R.J., Good, S.W., Kilpua, E.K.J.: 2020, On the radial and longitudinal variation of a magnetic cloud: ACE, wind, ARTEMIS and Juno observations. Solar Phys. 295(11), 157. DOI. ADS.

Desai, R.T., Zhang, H., Davies, E.E., Stawarz, J.E., Mico-Gomez, J., Iváñez-Ballesteros, P.: 2020, Threedimensional simulations of solar wind preconditioning and the 23 July 2012 interplanetary coronal mass ejection. Solar Phys. 295(9), 130. DOI. ADS.

dos Santos, L.F.G., Narock, A., Nieves-Chinchilla, T., Nuñez, M., Kirk, M.: 2020, Identifying flux rope signatures using a deep neural network. Solar Phys. 295(10), 131. DOI. ADS.

Dumbović, M., Vršnak, B., Guo, J., Heber, B., Dissauer, K., Carcaboso, F., Temmer, M., Veronig, A., Podladchikova, T., Möstl, C., Amerstorfer, T., Kirin, A.: 2020, Evolution of coronal mass ejections and the corresponding Forbush decreases: modeling vs. multi-spacecraft observations. Solar Phys. 295(7), 104. DOI. ADS.

El-Borie, M.A., El-Taher, A.M., Thabet, A.A., Bishara, A.A.: 2020, The interconnection between the periodicities of solar wind parameters based on the interplanetary magnetic field polarity $(1967-2018)$ : a cross wavelet analysis. Solar Phys. 295(9), 122. DOI. ADS.

Florido-Llinas, M., Nieves-Chinchilla, T., Linton, M.G.: 2020, Analysis of the helical kink stability of differently twisted magnetic flux ropes. Solar Phys. 295(9), 118. DOI. ADS. 
Giménez de Castro, C.G., Raulin, J.-P., Valio, A., Alaia, G., Alvarenga, V., Bortolucci, E.C., Fernandes, S.H., Francile, C., Giorgetti, T., Kudaka, A.S., López, F.M., Marcon, R., Marun, A., Zaquela, M.: 2020, HATS: a ground-based telescope to explore the THz domain. Solar Phys. 295(4), 56. DOI. ADS.

Hudson, H.S.: 2020, Solar flare build-up and release. Solar Phys. 295(10), 132. DOI. ADS.

Kostyuchenko, I., Bruevich, E.: 2021, The fine structure of the quasi-biennial oscillations of sunspot areas and the double magnetic cycle of the Sun. Solar Phys. 296(1), 8. DOI. ADS.

Lloveras, D.G., Vásquez, A.M., Nuevo, F.A., Mac Cormack, C., Sachdeva, N., Manchester, W., Van der Holst, B., Frazin, R.A.: 2020, Thermodynamic structure of the solar corona: tomographic reconstructions and MHD modeling. Solar Phys. 295(6), 76. DOI. ADS.

Melnik, V.N., Brazhenko, A.I., Konovalenko, A.A., Frantsuzenko, A.V., Yerin, S.M., Dorovskyy, V.V., Bubnov, I.M.: 2021, Properties of Type III and Type IIIb bursts in the frequency band of $8-80 \mathrm{MHz}$ during PSP perihelion at the beginning of April 2019. Solar Phys. 296(1), 9. DOI. ADS.

Molina, M.G., Dasso, S., Mansilla, G., Namour, J.H., Cabrera, M.A., Zuccheretti, E.: 2020, Consequences of a solar wind stream interaction region on the low latitude ionosphere: event of 7 October 2015. Solar Phys. 295(12), 173. DOI. ADS.

Morales, L.F., Santos, N.A.: 2020, Predicting extreme solar flare events using Lu and Hamilton avalanche model. Solar Phys. 295(11), 155. DOI. ADS.

Nandy, D.: 2021, Progress in solar cycle predictions: sunspot cycles 24-25 in perspective. Solar Phys. 296(3), 54. DOI. ADS.

Palacios, J., Utz, D., Hofmeister, S., Krikova, K., Gömöry, P., Kuckein, C., Denker, C., Verma, M., González Manrique, S.J., Campos Rozo, J.I., Koza, J., Temmer, M., Veronig, A., Diercke, A., Kontogiannis, I., Cid, C.: 2020, Magnetic flux emergence in a coronal hole. Solar Phys. 295(5), 64. DOI. ADS.

Ryabov, B.I., Vrublevskis, A.: 2020, Open-field coronal structures neighbouring the sunspot of AR 8535. Solar Phys. 295(1), 4. DOI. ADS.

Shanmugaraju, A., Pappa Kalaivani, P., Moon, Y.-J., Prakash, O.: 2021, Occurrence rate of radio-loud and halo CMEs in solar cycle 25: prediction using their correlation with the sunspot number. Solar Phys. 296(4), 75. DOI. ADS

Publisher's Note Springer Nature remains neutral with regard to jurisdictional claims in published maps and institutional affiliations. 